\title{
nature
}

biotechnology

\section{Do no harm}

\author{
Judicious choice of a target tumor antigen for adoptive T-cell therapy remains crucial not only for efficacy but also \\ for avoiding serious toxicities.
}

A doptive T-cell therapy (ACT) is now yielding promising data in Ahuman trials of advanced melanoma, lymphoma and several other difficult-to-treat cancers. It has taken decades to realize the goal of harnessing a patient's own T cells to seek and destroy tumor cells expressing particular antigens. But as newer-generation ACTs begin to generate impressive clinical data and attract breathless headlines, more researchers are entering the field and launching trials of their own. This means that now-more than ever-the field needs to take stock of how best to select the right $\mathrm{T}$-cell targeting antigen.

ACT involves isolating a patient's tumor-targeting T cells, activating and expanding them, and then injecting them back into the patient. Early trials used T lymphocytes isolated from resected tumors, but this worked well only for melanoma. To treat other types of tumors, researchers engineer T cells from patient peripheral blood to express high-affinity T-cell receptors (TCRs; which recognize antigenic peptides presented by polymorphic human leukocyte antigen (HLA) molecules) or chimeric antigen receptors (CARs; which comprise the variable regions of antibodies fused to TCR signaling components and recognize cell-surface proteins rather than peptide-HLA complexes).

Today, TCRs and CARs can be cloned from multiple sources. Targeted mutagenesis can further boost their affinity for the intended target tumor antigen. Rates of viral transduction of patient $\mathrm{T}$ cells routinely reach $80-90 \%$. Cells can be coaxed to proliferate extensively in vitro and engineered to express co-stimulatory molecules or cytokines to enhance effector function and/or anti-apoptotic proteins to prolong survival. And patients can be treated with ablative therapies to remove tumor-associated immunosuppressive cells that interfere with ACT.

And yet, the process of choosing an antigen present only in the tumor, and not elsewhere in the body, remains by no means straightforward-a fact borne out by several devastating and at times lethal toxicities seen in recent ACT trials.

In one case, $\mathrm{T}$ cells engineered to express a TCR recognizing a peptide from the tumor antigen MAGE-A3 presented by HLA-A ${ }^{*} 0201$ were injected into nine patients bearing a variety of metastatic MAGE-A3 ${ }^{+}$ tumors (J. Immunother. 36, 133-151, 2013). Within only a few days, three patients suffered neurological toxicities and two died; T cells were detected in their brains. In another case (Mol. Ther. 18, 843-851, 2010), after being injected with autologous $\mathrm{T}$ cells engineered to express an ERBB2-specific CAR, a patient with metastatic colon cancer suffered respiratory distress within just 15 minutes and died 5 days later; T cells were detected in her lungs.

Thanks to extensive follow-up work by the researchers involved, it is now clear investigators must carefully weigh in every trial whether sufficient information is known about patterns of expression of the intended target antigen, and its homologs, on healthy tissue. For example, MAGE-A3 was an appealing target in part because it is classified as a cancer-testes antigen, widely assumed to be absent from normal healthy adult tissues. However, follow-up work using quantitative PCR, NanoString and deep sequencing analysis revealed that a related cancer testes antigen MAGE-A12 was expressed in normal human brain tissue, potentially leading to the neurological toxicity observed.

In other cases, the issue may not be cross-reactivity with normal tissue antigens, but patient-to-patient variability. In some patients the presence of a low amount of target antigen in healthy tissues is not deleterious so long as a much higher amount of target antigen is expressed on the tumor. For other patients, it is deleterious. As yet, the quantitative limits of this 'window' of T-cell tolerance remain unknown.

Which raises the question: what practical steps can be taken to ameliorate these problems in future trials?

One solution may be to build a community database providing normal tissue antigen expression data as well as tools for identification of candidate HLA-binding peptides from candidate antigens and their close relatives. Such an effort would not be without difficulties. Highly activated $\mathrm{T}$ cells can often detect (and respond to) much lower amounts of antigen than can be detected by standard antibody-staining methods; individual cell types studied in isolation may express different antigens than the same cell types in intact three-dimensional tissues; and antigen presentation may differ markedly between individual patients-many of whom either have different single-nucleotide polymorphisms or may have been treated with drugs that modify epigenetic status (e.g., histone deacetylase inhibitors) that affect antigen expression in normal tissue.

A nearer-term step is for experienced researchers in the field to provide pointers for optimizing ACT trial design. Specifically, with regard to numbers of T cells injected, magnitude of T-cell effector functions, T-cell survival and TCR affinity for antigen, is more always better? At what stage does an engineered $\mathrm{T}$ cell become so potent that it recognizes and responds to not only the intended target tumor protein but also vanishingly small amounts of the same target on normal tissues? Could injection of lower numbers of T cells act as a pre-screen for unanticipated toxicities? Could such pilot injections be marked with magnetic nanoparticles for in vivo tracking? And more to the point, should T cells be engineered with inducible suicide mechanisms that can be rapidly deployed in the event of unexpected toxicity?

ACT is unlike any other cancer treatment. It can seek out cancer in places other therapies cannot. It can lead to memory $\mathrm{T}$ cells that persist in patients far longer than any other therapy, continuously eliminating residual cancer cells. And most importantly, it can induce dramatic complete remissions in patients for whom no other options exist. But with so many trials being initiated and the media spotlight on the field, it is imperative that investigators emphasize judicious target selection and design trials with a view to minimizing the potential for catastrophic adverse events. Failure to do so risks tarnishing ACT's considerable promise. 